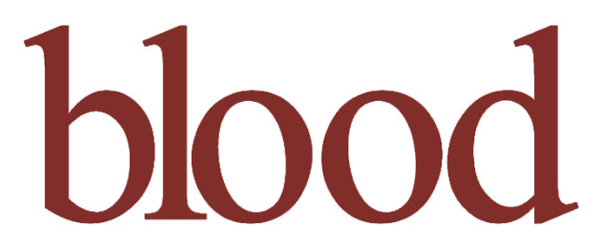

2005 106: 3658-3665

Prepublished online Aug 2, 2005;

doi:10.1182/blood-2005-03-1323

\title{
No evidence that FLT3 status should be considered as an indicator for transplantation in acute myeloid leukemia (AML): an analysis of 1135 patients, excluding acute promyelocytic leukemia, from the UK MRC AML10 and 12 trials
}

Rosemary E. Gale, Robert Hills, Panagiotis D. Kottaridis, Sivatharsini Srirangan, Keith Wheatley, Alan K. Burnett and David C. Linch

Updated information and services can be found at: http://bloodjournal.hematologylibrary.org/cgi/content/full/106/10/3658

Articles on similar topics may be found in the following Blood collections:

Transplantation (1286 articles)

Oncogenes and Tumor Suppressors (796 articles)

Clinical Trials and Observations (2398 articles)

Neoplasia (4075 articles)

Information about reproducing this article in parts or in its entirety may be found online at: http://bloodjournal.hematologylibrary.org/misc/rights.dtl\#repub_requests

Information about ordering reprints may be found online at:

http://bloodjournal.hematologylibrary.org/misc/rights.dtl\#reprints

Information about subscriptions and ASH membership may be found online at:

http://bloodjournal.hematologylibrary.org/subscriptions/index.dtl

Blood (print ISSN 0006-4971, online ISSN 1528-0020), is published semimonthly by the American Society of Hematology, 1900 M St, NW, Suite 200, Washington DC 20036.

Copyright 2007 by The American Society of Hematology; all rights reserved.

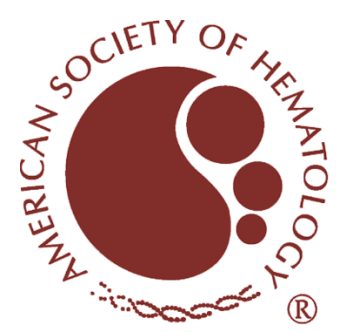




\section{No evidence that FLT3 status should be considered as an indicator for transplantation in acute myeloid leukemia (AML): an analysis of 1135 patients, excluding acute promyelocytic leukemia, from the UK MRC AML10 and 12 trials}

Rosemary E. Gale, Robert Hills, Panagiotis D. Kottaridis, Sivatharsini Srirangan, Keith Wheatley, Alan K. Burnett, and David C. Linch

Fetal liver tyrosine kinase 3 (FLT3) internal tandem duplications (ITDs) are powerful adverse prognostic indicators for relapse in acute myeloid leukemia (AML) but the most efficacious therapy for FLT3/ ITD ${ }^{+}$patients is currently unknown. We evaluated outcome according to FLT3/ITD status in 1135 adult patients treated according to United Kingdom Medical Research Council (UK MRC) AML protocols: 141 received an autograft, and 170 received a matched sibling allograft in first complete remission (CR). An FLT3/ITD was detected in $25 \%$ of patients and was an independent predictor for relapse $(P<.001)$. It remained prognostic for increased relapse in patients who received a transplant (odds ratio [OR] $=1.91 ; 95 \%$ confidence intervals $[\mathrm{Cls}]=1.13-3.21$; $\boldsymbol{P}=.02$ ), with no evidence of a difference in effect between patients who received an autograft $(\mathrm{OR}=2.39$; $\mathrm{Cls}=1.24-4.62)$ and patients who received an allograft (OR $=1.31$; $\mathrm{Cls}=0.56-3.06)$ (test for interaction, $P=.3$ ) or between patients who did or did not receive a transplant $(P=.4)$. These results were confirmed in an analysis of 186 patients randomized to receive or not receive an autograft in first $C R$ and in a donor-versus-no donor analysis of 683 patients to assess the role of allograft (for latter, FLT3/ITD- ${ }^{-}$OR $=0.70$, Cls $=0.53-0.92 ;$ FLT3/ITD $^{+}$OR $=0.59$, Cls $=0.40-0.87$; test for interaction, $P=.5)$. These results suggest that at present there is no strong evidence that FLT3 status should influence the decision to proceed to transplantation. (Blood. 2005;106:3658-3665)

() 2005 by The American Society of Hematology

\section{Introduction}

The use of more intensive induction regimens and better supportive care have led to major improvements in the complete remission (CR) rate in children and younger adults with acute myeloid leukemia (AML). A number of large multicenter studies have reported CR rates in younger adults in excess of $70 \%$. In the United Kingdom Medical Research Council (UK MRC) AML10 trial, the CR rate in adults between the ages of 15 and 60 years was $80 \%^{1}$ and was marginally higher still in the AML12 trial. ${ }^{2}$ The majority of patients still relapse and die of their disease, however, and attention over the past decade has been focused on postinduction strategies to consolidate remission. Three options have been extensively investigated. First, there are intensive consolidation chemotherapy regimens that do not require hematopoietic stem cell rescue, with high-dose cytosine arabinoside frequently used. ${ }^{3}$ Second, there are even more intensive chemotherapy or chemoradiotherapy consolidation regimens requiring autologous stem cell rescue. Third, there is the option of allogeneic transplantation for the younger patients with suitable donors.

A number of randomized trials have compared consolidation chemotherapy with autologous hematopoietic stem cell transplanta- tion (SCT) against standard-dose or no further chemotherapy, and the majority of these studies indicate a significantly lower relapse rate following the autograft. ${ }^{4}$ Autograft is associated, however, with a higher treatment-related mortality (TRM) and a lower salvage rate after subsequent relapse, so that no consistent overall survival (OS) benefit has been demonstrated. There is also no doubt that the relapse rate is markedly reduced following allogeneic transplantation, ${ }^{4}$ and analyses not taking into account time censoring $^{5}$ and other selection biases ${ }^{6}$ have made overly optimistic claims about patients' survival benefits. In multicenter trials analyzed on an intent-to-treat basis, however, the high toxicity has largely negated the impact of improved disease control on OS..$^{7-10}$

Despite the disappointment of transplantation results in most multicenter trials to date, the reduced relapse rate continues to provide the impetus for continuing studies of transplantation, particularly with a view to identifying subsets of patients who will benefit from a transplantation approach. Prognostic factors such as the karyotype of the leukemic cells and patient age have been used in this way. Most studies have shown that the presenting karyotype
From the Department of Haematology, Royal Free and University College Medical School, London, United Kingdom; Clinical Trials Unit, University of Birmingham, Birmingham, United Kingdom; Department of Haematology, University of Wales, Cardiff, Wales, on behalf of the National Cancer Research Institute (NCRI) Adult Leukaemia Working Party, United Kingdom.

Submitted March 31, 2005; accepted July 17, 2005. Prepublished online as Blood First Edition Paper, August 2, 2005; DOI 10.1182/blood-2005-03-1323.

P.D.K. was supported by the Leukaemia Research Fund of Great Britain. The DNA/RNA tissue bank is supported by the Kay Kendall Leukaemia Fund, the Leukaemia Research Fund of Great Britain, and the UK Medical Research Council.

Experimental analysis was performed by R.E.G., P.D.K., and S.S.; data analysis was performed by R.H.; statistical advice and interpretation were performed by K.W.; D.C.L. and A.K.B. contributed to the design of the study; and R.E.G. and D.C.L. wrote the manuscript with contributions from R.H., K.W., and A.K.B.

\section{An Inside Blood analysis of this article appears at the front of this issue.}

Reprints: Rosemary E. Gale, Dept of Haematology, Royal Free and University College Medical School, 98 Chenies Mews, London WC1E 6HX, United Kingdom; e-mail: rosemary.gale@ucl.ac.uk.

The publication costs of this article were defrayed in part by page charge payment. Therefore, and solely to indicate this fact, this article is hereby marked "advertisement" in accordance with 18 U.S.C. section 1734.

(C) 2005 by The American Society of Hematology 
predicts for relapse after transplantation in patients who actually receive a transplant. ${ }^{11-14}$ However, the results in prospective intent-to-treat analyses have been inconsistent and there is uncertainty as to which risk groups are most likely to benefit from transplantation. Whereas the South West Oncology Group concluded that patients with favorable cytogenetics benefited from transplantation, ${ }^{14}$ the opposite conclusion was drawn from the UK MRC AML10 and 12 trials. ${ }^{10,15}$ In those patients with adverse cytogenetics, it has been suggested that the outcome is improved by allogeneic but not autologous transplantation, ${ }^{14,16}$ but this was not apparent in the UK MRC AML10 trial. ${ }^{10}$ The outcome for patients with intermediate risk cytogenetics is similarly inconsistent between trials. ${ }^{17}$

Within the past few years the presence of an activating internal tandem duplication (ITD) in the juxtamembrane domain of the tyrosine kinase receptor gene fetal liver tyrosine kinase 3 (FLT3) has been identified as a powerful prognostic indicator predicting for relapse from CR. ${ }^{18-20}$ For example, in our own study of 854 patients entered into the UK MRC AML10 and 12 trials, multivari- able analysis demonstrated that an FLT3/ITD was the most important factor, ahead of cytogenetics, predicting for relapse from $\mathrm{CR}$ and disease-free survival (DFS) $(P<.001) .{ }^{21}$ Furthermore, the incidence of an FLT3/ITD was significantly higher in patients with a normal karyotype, the major component of the intermediate cytogenetics group, than in those with abnormal cytogenetics $(34 \%$ versus $21 \%$, respectively; $P<.001) .{ }^{21}$ Activating point mutations also occur in the second tyrosine kinase domain of FLT3 in approximately $7 \%$ of AML patients, but for reasons that are not yet clear, these are probably not indicative of a poor outcome. ${ }^{18,19}$

It is possible that the more intensive therapy used in an autograft could overcome the poor prognosis associated with an FLT3/ITD. Furthermore, allograft procedures introduce not only intensified therapy but also a graft-versus-leukemia effect, and it is conceivable that the characteristics of leukemic blasts containing an FLT3/ITD, which impart resistance to chemotherapy and possibly chemoradiotherapy, would not cause cross-resistance to immune-mediated cytotoxicity. We have therefore examined the outcome according to FLT3/ITD status of 1135 adult patients with AML entered into the UK MRC AML10 or 12

Table 1. Clinical and demographic details of 1135 AML patients in total cohort

\begin{tabular}{|c|c|c|c|c|c|}
\hline & Total & FLT3/ITD- (\% total ITD-) & FLT3/ITD+ $\left(\%\right.$ total ITD $\left.{ }^{+}\right)$ & $\%$ FLT3/ITD + & $P$ \\
\hline Total & 1135 & 852 & 283 & 25 & - \\
\hline AML10 & 428 & 329 (39) & 99 (35) & 23 & .3 \\
\hline AML12 & 707 & $523(61)$ & $184(65)$ & 26 & - \\
\hline De novo & 1042 & 778 (91) & $264(93)$ & 25 & .5 \\
\hline Secondary & 92 & $73(9)$ & $19(7)$ & 21 & - \\
\hline Unknown & 1 & 1 & 0 & 0 & - \\
\hline FAB type & & & & & .001 \\
\hline MO & 36 & $34(4)$ & $2(1)$ & 6 & \\
\hline M1 & 222 & $159(20)$ & $63(23)$ & 28 & \\
\hline M2 & 343 & $264(33)$ & $79(29)$ & 23 & \\
\hline M4 & 278 & $194(24)$ & $84(31)$ & 30 & \\
\hline M5 & 128 & $91(11)$ & $37(14)$ & 29 & \\
\hline M6 & 32 & $29(4)$ & $3(1)$ & 9 & \\
\hline M7 & 16 & $15(2)$ & $1(<0.5)$ & 6 & \\
\hline RAEB-t & 20 & $18(2)$ & $2(1)$ & 10 & \\
\hline Bilineage & 1 & 0 & $1(<0.5)$ & 100 & \\
\hline Other & 1 & $1(<0.5)$ & 0 & 0 & \\
\hline Unknown & 58 & 47 & 11 & 19 & \\
\hline Sex & & & & & .4 \\
\hline Male & 568 & $432(51)$ & $136(48)$ & 24 & \\
\hline Female & 567 & $420(49)$ & $147(52)$ & 26 & \\
\hline Age, y & & & & & .14 \\
\hline $15-29$ & 230 & $179(21)$ & $51(18)$ & 22 & \\
\hline 30-39 & 248 & $188(22)$ & $60(21)$ & 24 & \\
\hline $40-49$ & 333 & $247(29)$ & $86(30)$ & 26 & \\
\hline $50-59$ & 305 & $228(27)$ & 77 (27) & 25 & \\
\hline 60 or older & 19 & $10(1)$ & $9(3)$ & 47 & \\
\hline Median & 42 & 42 & 43 & - & \\
\hline WBC, $\times 10^{9} / \mathrm{L}$ & & & & & $<.001$ \\
\hline Less than 10 & 358 & $318(38)$ & $40(14)$ & 11 & \\
\hline 10-19.9 & 181 & $141(17)$ & $40(14)$ & 22 & \\
\hline $20-49.9$ & 238 & 177 (21) & 61 (22) & 26 & \\
\hline $50-99.9$ & 169 & 109 (13) & $60(21)$ & 36 & \\
\hline 100 or above & 168 & $89(11)$ & $79(28)$ & 47 & \\
\hline Unknown & 21 & 18 & 3 & 14 & \\
\hline Median & 21 & 16 & 50 & - & \\
\hline Cytogenetics & & & & & $<.001$ \\
\hline Favorable & 151 & $134(19)$ & $17(8)$ & 11 & \\
\hline Intermediate & 655 & 455 (65) & $200(89)$ & 31 & \\
\hline Adverse & 116 & $108(15)$ & $8(4)$ & 7 & \\
\hline Unknown & 213 & 155 & 58 & 27 & \\
\hline
\end{tabular}

$P$ values are for Mantel-Haenszel test for trend in age and WBC count and for chi-square test for heterogeneity elsewhere.

FAB indicates French-American-British; RAEB-t, refractory anemia with excess of blasts in transformation; WBC, white blood cell count; and —, not applicable. 
From www.bloodjournal.org at UCL Library Services on August 20, 2008. For personal use only.

Table 2. Demographic details of 311 AML patients who received a transplant in first complete remission

\begin{tabular}{|c|c|c|c|c|c|c|c|c|c|c|}
\hline \multirow[b]{2}{*}{ FLT3 status } & \multicolumn{5}{|c|}{ Allograft } & \multicolumn{5}{|c|}{ Autograft } \\
\hline & Total & ITD $^{-}$ & ITD ${ }^{+}$ & $\%$ ITD $^{+}$ & $P$ & Total & ITD- & ITD $^{+}$ & $\%$ ITD $^{+}$ & $P$ \\
\hline Total & 170 & 135 & 35 & 21 & - & 141 & 104 & 37 & 26 & - \\
\hline AML10 & 68 & 55 & 13 & 19 & $.7^{\star}$ & 59 & 48 & 11 & 19 & $.08^{*}$ \\
\hline AML12 & 102 & 80 & 22 & 22 & & 82 & 56 & 26 & 32 & \\
\hline De novo & 160 & 127 & 33 & 21 & $1.0 \dagger$ & 136 & 99 & 37 & 27 & $.17 \dagger$ \\
\hline Secondary & 10 & 8 & 2 & 20 & & 5 & 5 & 0 & 0 & \\
\hline FAB type & & & & & .5 & & & & & .4 \\
\hline MO & 9 & 9 & 0 & 0 & & 4 & 4 & 0 & 0 & \\
\hline M1 & 29 & 22 & 7 & 24 & & 27 & 17 & 10 & 37 & \\
\hline M2 & 41 & 32 & 9 & 22 & & 36 & 24 & 12 & 33 & \\
\hline M4 & 46 & 34 & 12 & 26 & & 38 & 30 & 8 & 21 & \\
\hline M5 & 20 & 16 & 4 & 20 & & 23 & 19 & 4 & 17 & \\
\hline M6 & 9 & 9 & 0 & 0 & & 4 & 3 & 1 & 25 & \\
\hline M7 & 3 & 2 & 1 & 33 & & 0 & 0 & 0 & 0 & \\
\hline RAEB-t & 5 & 4 & 1 & 20 & & 3 & 3 & 0 & 0 & \\
\hline Unknown & 8 & 7 & 1 & 13 & & 6 & 4 & 2 & 33 & \\
\hline Sex & & & & & .3 & & & & & .5 \\
\hline Male & 85 & 65 & 20 & 24 & & 70 & 50 & 20 & 29 & \\
\hline Female & 85 & 70 & 15 & 18 & & 71 & 54 & 17 & 24 & \\
\hline Age, y & & & & & 6 & & & & & 1.0 \\
\hline $15-29$ & 50 & 43 & 7 & 14 & & 36 & 25 & 11 & 31 & \\
\hline $30-39$ & 62 & 46 & 16 & 26 & & 34 & 27 & 7 & 21 & \\
\hline $40-49$ & 48 & 37 & 11 & 23 & & 40 & 29 & 11 & 28 & \\
\hline $50-59$ & 10 & 9 & 1 & 10 & & 29 & 23 & 6 & 21 & \\
\hline 60 or older & 0 & 0 & 0 & 0 & & 2 & 0 & 2 & 100 & \\
\hline Median & 35 & 35 & 35 & - & & 40 & 40 & 41 & - & \\
\hline Cytogenetics & & & & & .9 & & & & & .8 \\
\hline Favorable & 16 & 13 & 3 & 19 & & 1 & 1 & 0 & 0 & \\
\hline Intermediate & 99 & 78 & 21 & 21 & & 106 & 79 & 27 & 25 & \\
\hline Adverse & 17 & 13 & 4 & 24 & & 4 & 3 & 1 & 25 & \\
\hline Unknown & 38 & 31 & 7 & 18 & & 30 & 21 & 9 & 30 & \\
\hline
\end{tabular}

$P$ values are for Mantel-Haenszel test for trend in age and for chi-square test for heterogeneity elsewhere.

- indicates not applicable.

${ }^{\star} P$ value for the difference in $\% \mathrm{ITD}^{+}$patients in the AML10 and AML12 studies.

$\dagger P$ value for the difference in \%ITD ${ }^{+}$patients with de novo or secondary AML.

trials, of whom 141 received an autograft and 170 received an allograft from a matched sibling donor in first remission. In an attempt to ascertain whether SCT is likely to modify the outcome in patients with an FLT3/ITD, we have analyzed outcome according to FLT3 status in 186 patients randomized to receive or not receive consolidation with an autologous stem cell transplant. In addition, to further evaluate the possible benefit of an allograft in FLT3/ITD $^{+}$patients, we have performed a donor-versus-no donor analysis in the 683 patients in whom the FLT3/ITD status was available.

\section{Patients, materials, and methods}

\section{Patients}

DNA or complementary DNA (cDNA) was available from blast cells of 1135 AML patients, excluding acute promyelocytic leukemia (APL), entered into either the UK MRC AML10 $(n=428)$ or AML12 $(n=707)$ trials. The majority of patients (1042 of $1134,92 \%)$ had de novo AML. Median age at entry was 42 years and only 19 patients were aged 60 or older. Patient details are given in Table 1. Outcome data were available for all 1135 patients. The remission rate was $85 \%$, and a total of $340(35 \%)$ underwent transplantation in first CR, of whom 170 received a matched sibling allograft and 141 received an autograft. Details of these patients are given in Table 2. Twenty-nine patients received other types of transplant (eg, from a matched unrelated donor), and these patients are not discussed further because of the lack of statistical reliability with such small numbers.

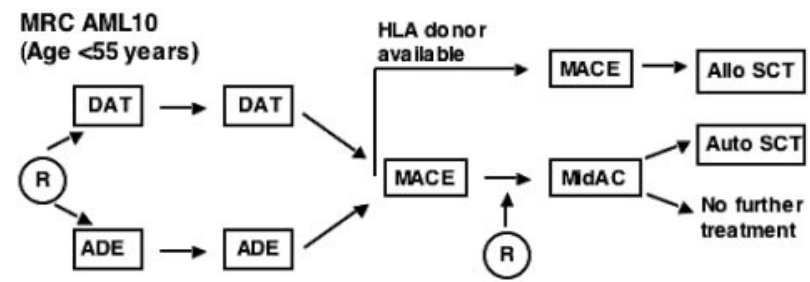

MRC AML12

(Age $<60$ years)

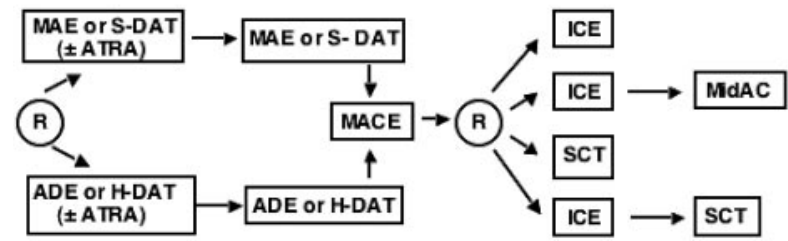

Figure 1. Outline of the relevant treatment protocols for patients in MRC trials AML10 and 12. More extensive details on the protocols are as published. ${ }^{1,2,21}$ DAT indicates daunorubicin + AraC + 6-thioguanine; ADE, AraC + daunorubicin + etoposide; HLA, human leukocyte antigen; MACE, amsacrine + AraC + etoposide; MidAC, mitoxantrone + AraC; MAE, mitoxantrone + AraC + etoposide; ATRA, all trans retinoic acid; ICE, idarubicin + cytosine arabinoside + etoposide; R, randomization; and SCT, stem cell transplantation. In AML12, either a standard $(\mathrm{S})$ or high $(\mathrm{H})$ dose of AraC was given. Patients in AML12 received an allogeneic transplant if a suitable matched sibling donor was available or, if no donor was available, an autologous transplant. 
Figure 2. Clinical outcome in the total cohort of 1135 patients according to FLT3/ITD status. (A) Relapse risk. (B) Overall survival. Obs indicates observed; Exp, expected.

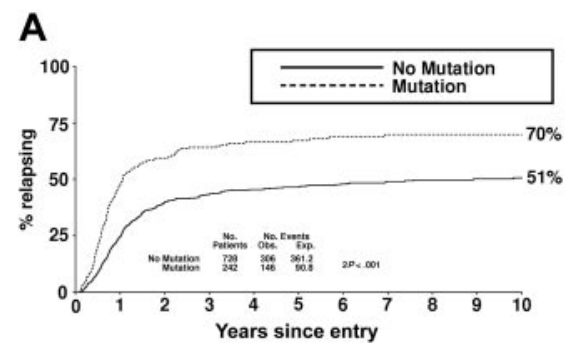

B

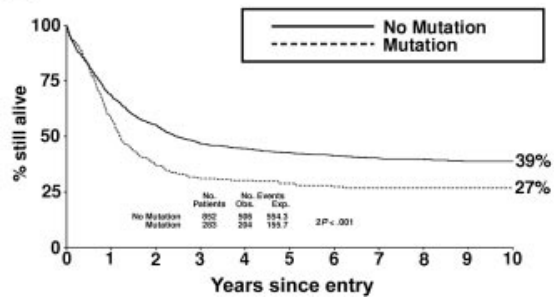

Approval for these studies was obtained from the Multi-Centre Research Ethics Committee for Wales. Informed consent was provided in accordance with the Declaration of Helsinki.

\section{Therapy}

Details of randomization and treatment regimens for patients entered into UK MRC AML10 or AML12 trials have been published elsewhere ${ }^{1,2,21}$ and are outlined in the flowcharts (Figure 1).

\section{End points}

$\mathrm{CR}$ was defined as a normocellular bone marrow (BM) containing less than $5 \%$ blasts and showing evidence of normal maturation of other marrow elements. Peripheral blood regeneration was not a requirement, but $97 \%$ of cases defined as CR achieved a neutrophil count of $1 \times 10^{9} / \mathrm{L}$ and a platelet count of $100 \times 10^{9} / \mathrm{L}$. Remission failures were classified by the clinicians as either partial remission (defined as $5 \%-15 \%$ blasts or $<5 \%$ blasts but a hypocellular BM), resistant disease ( $>15 \%$ blasts in the BM), or induction death (ie, related to treatment or hypoplasia). Where the clinician's evaluation was not available, deaths within 30 days of entry were classified as induction death and all other failures to achieve remission as resistant disease. OS was defined as the time from entry to death. For patients achieving CR, DFS was the time from the date of first CR to an event (death in first CR or relapse) and relapse risk (RR) was the cumulative probability of relapse, censoring at death in $\mathrm{CR}$.

\section{PCR analysis of the FLT3/ITD mutation}

Exons 14 and 15 (previously designated 11 and 12) and the intervening intron of the FLT3 gene were amplified from DNA or cDNA as previously described..$^{21}$ Any patient with an additional higher-molecular weight band was considered to be positive for an FLT3/ITD $\left(\right.$ FLT3/ITD $\left.^{+}\right)$, irrespective of the size of the band or relative level of mutant. The presence and quantification of a mutation was confirmed by polymerase chain reaction (PCR) amplification with a fluorescently labeled primer followed by fragment analysis on the CEQ 8000 DNA Genetic Analysis System (Beckman Coulter, Fullerton, CA).

\section{Statistical methods}

The Wilcoxon 2-sample test (for continuous data), Mantel-Haenszel test for trend (for ordinal data), and the chi-square test (for heterogeneity) were used to test for differences in clinical and demographic data by FLT3/ITD positivity. Kaplan-Meier life tables were constructed for survival data and were compared by means of the log-rank test, with surviving patients being censored at April 1, 2004. Follow-up was up to date for the vast majority of patients, and the small number of patients lost to follow-up are censored at the date they were last known to be alive. Median follow-up was 7.5 years (range, 2-16 years). Analysis of time-to-event data was done using standard log-rank methods, and odds ratio (OR) plots, with tests for heterogeneity, were used to investigate whether the prognostic relevance of FLT3/ITD differed between treatment subgroups. Multivariable logistic regression analysis was used to find the factors most closely associated with CR rate, and multivariable Cox models were used to analyze OS, DFS, and RR. Models were fitted using forward selection, with variables added to the model if they reached significance at the $P=.01$ level. Because of multiple testing, the level of significance was set at $P=.01$ for all tests. All $P$ values are 2 tailed.

\section{Results}

\section{Frequency of an FLT3/ITD and impact on overall outcome}

An FLT3/ITD was detected in 283 of 1135 non-APL AML patients (25\%) treated according to the MRC AML10 and 12 trial protocols (Table 1). Data on 854 patients including APL were reported previously, ${ }^{21}$ and a similar incidence was observed (227 of 854, 27\%). The distribution of an FLT3/ITD in the different cytogenetic risk groups was also similar: $11 \%$ of patients with favorable

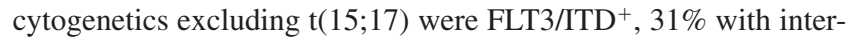
mediate cytogenetics, and $7 \%$ with adverse cytogenetics. The incidence of FLT3/ITD positivity was similar $(P=.2)$ among patients who received an autograft (37 of 141, 26\%) and those receiving a matched sibling donor allograft (35 of 170, 21\%; Table 2). Clinical investigators were not aware of the FLT3 status of patients and so did not direct treatment on that basis. Samples for quantification were available in 69 of the 72 transplant recipients who were FLT3/ITD ${ }^{+}$(37 autografts and 32 allografts). The median mutant level for all patients was $37 \%$ of total FLT3 (range, 4\%-96\%) and did not differ between autografts (median, 39\%; range, $4 \%-88 \%$ ) and allografts (median, 35\%; range, 5\%-96\%). Thirteen of the FLT3/ITD ${ }^{+}$autograft patients $(35 \%)$ and 7 of the FLT3/ITD $^{+}$allograft patients $(22 \%)$ had at least $45 \%$ mutant FLT3/ITD. Nine autograft and 5 allograft patients had more than 1 mutant FLT3/ITD.

Table 3. Clinical outcome for transplant recipients at 5 years from time of transplantation

\begin{tabular}{|c|c|c|c|c|c|c|c|c|}
\hline & \multicolumn{3}{|c|}{ Allograft } & \multicolumn{3}{|c|}{ Autograft } & \multicolumn{2}{|c|}{ Overall } \\
\hline & ITD $^{-}$ & ITD $^{+}$ & OR (95\% Cls) & ITD $^{-}$ & $\mathrm{ITD}^{+}$ & OR (95\% Cls) & OR (95\% Cls) & Het $P^{*}$ \\
\hline Patients & 135 & 35 & - & 104 & 37 & - & - & - \\
\hline TRM & $27 \%$ & $40 \%$ & $1.48(0.72-3.06)$ & $21 \%$ & $15 \%$ & $0.77(0.29-2.08)$ & $1.18(0.66-2.12)$ & .3 \\
\hline $\mathrm{RR}$ & $25 \%$ & $31 \%$ & $1.31(0.56-3.06)$ & $35 \%$ & $56 \%$ & $2.39(1.24-4.63)$ & $1.91(1.13-3.21)$ & .3 \\
\hline DFS & $55 \%$ & $41 \%$ & $1.41(0.82-2.44)$ & $51 \%$ & $38 \%$ & $1.69(0.98-2.93)$ & $1.54(1.05-2.28)$ & .6 \\
\hline os & $58 \%$ & $44 \%$ & $1.44(0.81-2.54)$ & $54 \%$ & $43 \%$ & $1.57(0.89-2.77)$ & $1.51(1.01-2.25)$ & .8 \\
\hline
\end{tabular}

OR indicates odds ratio; Cls, confidence intervals; TRM, treatment-related mortality; RR, relapse risk; DFS, disease-free survival; OS, overall survival; - , not applicable.

${ }^{*}$ Heterogeneity $P$ values (Het $P$ ) are for the interaction between type of transplant and FLT3/ITD status. 

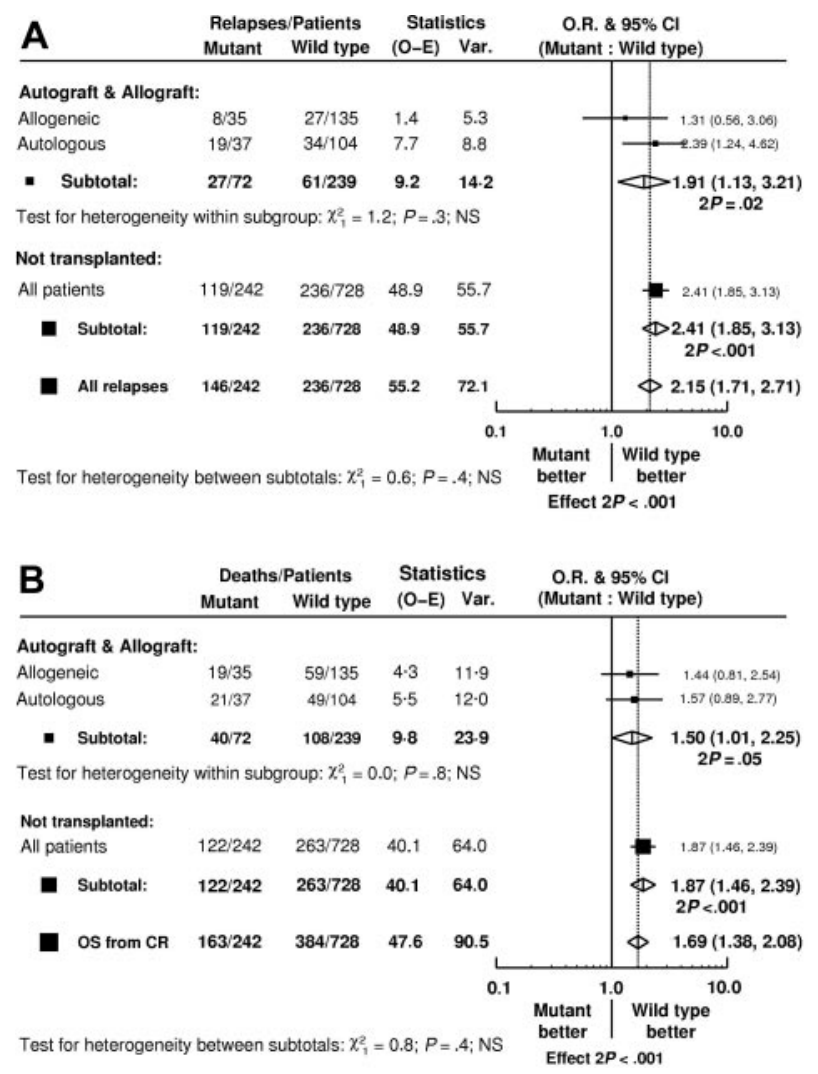

Figure 3. Mantel-Byar analysis of clinical outcome according to FLT3/TD status in 970 patients who achieved complete remission. All patients begin in the no transplant group but are censored at the point of transplantation. (A) Relapse rate. (B) Overall survival. O-E indicates observed - expected; Var., variance; NS, not significant.

\section{CR rate}

The CR rate for this cohort of 1135 younger adult patients with non-APL AML was $85 \%$, with no difference between those who were FLT3/ITD ${ }^{+}$and those who were FLT3/ITD ${ }^{-}(86 \%$ and $85 \%$, respectively). The frequencies of resistant disease and induction death in the FLT3/ITD ${ }^{+}$and FLT3/ITD ${ }^{-}$patients were also not significantly different $(9 \%$ and $9 \%$ for resistant disease, respectively, $6 \%$ and $6 \%$ for induction death). These results are not significantly different from those in all patients who entered into the UK MRC AML10 and 12 trials (data not shown).

\section{RR and DFS}

For the whole cohort of 970 patients who achieved a CR, RR at 10 years was $70 \%$ in those patients who were FLT3/ITD ${ }^{+}$compared with $51 \%$ in those who were FLT3/ITD $^{-}(\mathrm{OR}=2.15,95 \%$ confidence intervals $[\mathrm{CIs}]=1.71-2.71 ; P<.001$; Figure 2A).
Similarly, DFS at 10 years was $23 \%$ in FLT3/ITD ${ }^{+}$compared with $37 \%$ in FLT3/ITD ${ }^{-}$patients $(\mathrm{OR}=1.73 ; 95 \% \mathrm{CIs}=1.42-2.10$; $P<.001)$. On multivariable analysis, considering FLT3 status, age, presentation white cell count, de novo or secondary AML, cytogenetic risk group, and response to the first cycle of induction chemotherapy as candidate variables, FLT3 status remained a major independent predictor of relapse $(P<.001)$. Cytogenetic risk group was also highly predictive $(P<.001)$.

\section{OS}

OS at 10 years from diagnosis for the total cohort of 1135 patients was $27 \%$ and $39 \%$ for those who were FLT3/ITD ${ }^{+}$and FLT3/ITD ${ }^{-}$, respectively $(\mathrm{OR}=1.49 ; 95 \% \mathrm{CIs}=1.25-1.78 ; P<.001$; Figure 2B). In multivariable analysis, the presence of an FLT3/ITD was an independent risk factor for survival $(P<.001)$, although less important than cytogenetics and age $(P<.001$ in each case).

\section{The impact of FLT3 status on outcome following transplantation}

Of the 141 patients who received an autograft, 37 had an FLT3/ITD mutation and 104 did not. Of the 170 patients who received an allograft, 35 had an FLT3/ITD and 135 did not. In neither group, nor overall for all patients who received a transplant, was there evidence of any difference in the TRM between those with and without an FLT3/ITD (Table 3). Among the total group of 311 patients who received a transplant, FLT3/ITD was associated with a greater $\mathrm{RR}(\mathrm{OR}=1.91 ; 95 \% \mathrm{CIs}=1.13-3.21)$ in line with the increased risk among patients who did not receive a transplant (Figure 3A). There was no evidence of heterogeneity of effect between those patients who received an autograft (Figure 4A) and those who received an allograft (Figure 4C, 3A). In the patients who received an autograft, the RR at 5 years was $56 \%$ versus $35 \%$ in the FLT3/ITD ${ }^{+}$and FLT3/ITD ${ }^{-}$patients, respectively (Table 3). In the patients who received an allograft, RR was $31 \%$ versus $25 \%$, respectively, with wide confidence intervals due to the relatively small number of patients. The adverse impact of an FLT3/ITD on DFS was unaffected by type of transplant (Table 3). Likewise, the adverse effect of an FLT3/ITD on OS from first CR was not moderated by transplant (test for heterogeneity $P=.4$ ) or by type of transplant (test for heterogeneity $P=.8$ ); the OR among patients who received a transplant was $1.50(95 \% \mathrm{CIs}=1.01-2.25) \mathrm{com}-$ pared with an OR for the entire population of remitters of 1.69 $(95 \%$ CIs $=1.38-2.08$; Figure 3B).

Within patients who received a transplant, a higher level of mutant FLT3/ITD was associated with increased RR ( $P=.003$ for trend). There was no evidence of any heterogeneity of effect by type of transplant $(P=.6)$, although only a small number of patients had a high level of mutant, making it difficult to draw any firm conclusions. Within the patients who received an autograft, 9 of the 13 with at least $45 \%$
A

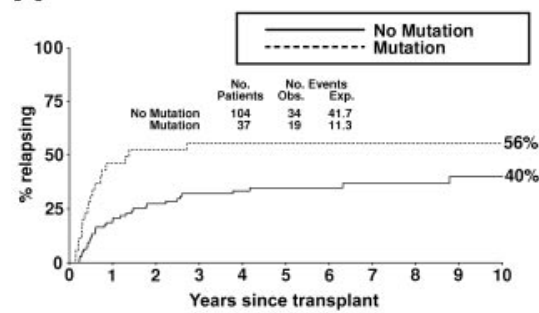

B

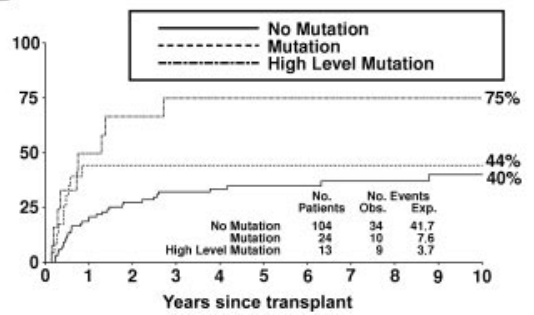

C

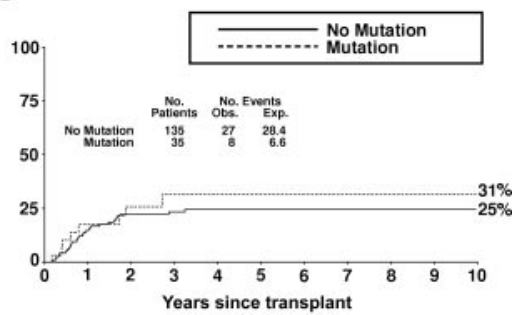

Figure 4. Relapse rate according to FLT3/ITD status in patients who received a transplant in first complete remission. (A) Patients receiving an autograft. (B) Patients who received an autograft with low-level (<45\%) or high-level ( $\geq 45 \%)$ mutant FLT3/ITD. (C) Patients receiving a matched sibling allograft. Obs indicates observed; Exp, expected. 
From www.bloodjournal.org at UCL Library Services on August 20, 2008. For personal use only.

BLOOD, 15 NOVEMBER $2005 \cdot$ VOLUME 106, NUMBER 10

Table 4. Clinical outcome at 5 years for 186 patients randomized to receive or not receive an autologous stem cell transplant

\begin{tabular}{|c|c|c|c|c|c|c|c|c|}
\hline & \multicolumn{3}{|c|}{ FLT3/ITD- } & \multicolumn{3}{|c|}{ FLT3/ITD ${ }^{+}$} & \multicolumn{2}{|c|}{ Overall } \\
\hline & SCT & No SCT & OR (95\% Cl) & SCT & No SCT & OR (95\% Cl) & OR $(95 \% \mathrm{Cl})$ & Het $P^{*}$ \\
\hline Patients & 68 & 57 & - & 35 & 26 & - & - & - \\
\hline TRM & $27 \%$ & $4 \%$ & $6.99(1.16-4.21)$ & $19 \%$ & $18 \%$ & $0.99(0.35-2.80)$ & $1.62(0.66-3.99)$ & .06 \\
\hline $\mathrm{RR}$ & $40 \%$ & $50 \%$ & $0.69(0.40-1.18)$ & $59 \%$ & $89 \%$ & $0.41(0.21-0.80)$ & $0.56(0.37-0.86)$ & .2 \\
\hline DFS & $49 \%$ & $48 \%$ & $0.96(0.59-1.55)$ & $33 \%$ & $8 \%$ & $0.45(0.25-0.83)$ & $0.72(0.49-1.05)$ & .06 \\
\hline os & $55 \%$ & $57 \%$ & $1.05(0.64-1.73)$ & $48 \%$ & $8 \%$ & $0.40(0.21-0.76)$ & $0.73(0.49-1.08)$ & .02 \\
\hline
\end{tabular}

${ }^{*}$ Heterogeneity $P$ values (Het $P$ ) are for the interaction between transplant or no transplant and FLT3/ITD status.

OR indicates odds ratio; $\mathrm{Cl}$, confidence interval; TRM, treatment-related mortality; RR, relapse risk, DFS, disease-free survival; OS, overall survival; and -, not applicable.

mutant FLT/ITD relapsed, giving a RR of $75 \%$ at 5 years (Figure 4B); for patients who received an allograft, only 7 patients had a high level of mutant (with 2 relapses).

\section{Autologous SC transplant randomization}

Of the patients in these trials where the FLT3/ITD status was known, 186 entered prospective randomizations to receive $(n=103)$ or not receive $(\mathrm{n}=83)$ an autologous $\mathrm{SC}$ transplant. Thirty-five of the 103 patients $(34 \%)$ randomized to receive an autograft were FLT3/ITD $^{+}, 26$ of the $83(31 \%)$ randomized to not receive the transplant were $\mathrm{FLT}_{3} / \mathrm{ITD}^{+}$. Of the 103 patients randomized to an autograft, 80 received transplants. Randomization to an autograft was associated with a decreased RR (overall OR $=0.56 ; 95 \%$ CI $=0.37-0.86 ; P=.008)$. This was the case in both the FLT3/ITD $^{-}$patients (RR $40 \%$ SCT versus $50 \%$ no SCT) and FLT3/ITD $^{+}$patients (59\% versus $89 \%$ ), and testing for heterogeneity showed no difference in the effect of transplantation according to FLT3/ITD status $(P=.2$; Table 4$)$. However, the beneficial effect of SCT on relapse did not translate into an overall significant survival benefit (overall OR for OS $=0.73 ; 95 \%$ CIs $=0.49-1.08$; $P=.12$ ), although there was some evidence of possible heterogeneity in survival between transplantation and FLT3/ITD status (OS for $\mathrm{FLT3}_{/ \mathrm{ITD}^{-}}=55 \%$ SCT versus $57 \%$ no SCT; for FLT3/ $\mathrm{ITD}^{+}=48 \%$ SCT versus $8 \%$ no SCT; $P=.02$; Table 4 ).

\section{Donor-versus-no donor comparison}

In 683 patients in whom the FLT3/ITD status was known, 273 had a matched sibling donor available. There was no difference in the characteristics of the patients with and without a donor (data not shown). Sixty-eight of the patients with a donor available had an FLT3/ITD (25\%) of whom 50 received a transplant, 37 of them in first CR, and 114 of the 410 patients without a donor had an FLT3/ITD (28\%). There were 205 FLT3/ITD $^{-}$patients with a donor, 160 of whom received a transplant, 136 of them in first CR, and 296 FLT3/ITD $^{-}$patients without a donor. On the donorversus-no donor analysis, the risk of relapse at 5 years was reduced in the donor group in both the FLT3/ITD ${ }^{+}(50 \%$ donor versus $75 \%$ no donor) and FLT3/ITD $^{-}(30 \%$ versus $51 \%)$ cases as well as overall, but OS was not significantly improved (FLT3/ITD ${ }^{+} 43 \%$ versus $31 \%$; FLT3/ITD $^{-} 54 \%$ versus $49 \%$; overall $P=.12$; Table 5). Testing for heterogeneity showed no significant differences in outcome depending on the presence of a donor in patients who were FLT3/ITD ${ }^{+}$compared with those who were FLT3/ITD ${ }^{-}$ for any of the end points (Table 5; Figure 5).

\section{Discussion}

This study of the prognostic impact of an FLT3/ITD in 1135 younger patients with AML indicates that its presence in the leukemic blasts is a major predictor of relapse from $\mathrm{CR}$ and, ultimately, of overall survival. The results extend our previously reported data in a smaller number of patients ${ }^{21}$ and are similar to those obtained from most other cohort studies. ${ }^{18-20}$ The collective data imply that the presence of an FLT3/ITD is associated with chemoresistance in the leukemic stem cell. The present analysis was therefore designed to ascertain whether the adverse prognosis associated with an FLT3/ITD also negatively impacts on the outcome of either autologous or allogeneic transplantation. To do this, outcome of patients who received a transplant was compared according to FLT3 status. Such an analysis can only indicate whether FLT3 retains its prognostic relevance following SCT; it can say nothing about whether SCT modifies the poor prognosis of FLT/ITD $^{+}$patients. To investigate the latter, randomized comparisons of SCT versus not with subgroup analysis by FLT3 status are needed. Thus, the data from 2 randomized comparisons (autograft versus not and a donor-versus-no donor genetic randomization) were used to investigate whether the important adverse factor of FLT3/ITD positivity could be overcome either by the chemotherapy dose escalation employed in an autograft consolidation procedure or by an allograft that additionally recruits a graft-versusleukemia effect. It is noteworthy that the conditioning regimen of the UK MRC trials used in both the autografts and allografts was the same, namely cyclophosphamide $120 \mathrm{mg} / \mathrm{kg}$ over 2 days followed by total body irradiation given either as a single fraction

Table 5. Donor-versus-no donor analysis of clinical outcome at 5 years after remission in 683 patients

\begin{tabular}{|c|c|c|c|c|c|c|c|c|}
\hline & \multicolumn{3}{|c|}{ FLT3/ITD- } & \multicolumn{3}{|c|}{ FLT3/ITD $^{+}$} & \multicolumn{2}{|c|}{ Overall } \\
\hline & Donor & No donor & OR (95\% Cl) & Donor & No donor & OR (95\% Cl) & OR (95\% Cl) & Het $P^{*}$ \\
\hline Patients & 205 & 296 & - & 68 & 114 & - & - & - \\
\hline TRM & $23 \%$ & $16 \%$ & $1.60(1.03-2.49)$ & $32 \%$ & $11 \%$ & $2.91(1.22-6.97)$ & $1.81(1.22-2.68)$ & .2 \\
\hline RR & $30 \%$ & $51 \%$ & $0.70(0.53-0.92)$ & $50 \%$ & $75 \%$ & $0.59(0.40-0.87)$ & $0.66(0.53-0.83)$ & .5 \\
\hline DFS & $46 \%$ & $41 \%$ & $0.88(0.70-1.11)$ & $34 \%$ & $22 \%$ & $0.77(0.54-1.09)$ & $0.84(0.69-1.02)$ & .5 \\
\hline os & $54 \%$ & $49 \%$ & $0.90(0.71-1.16)$ & $43 \%$ & $31 \%$ & $0.75(0.52-1.09)$ & $0.85(0.70-1.05)$ & .4 \\
\hline
\end{tabular}

*Heterogeneity $P$ values (Het $P$ ) are for the interaction between donor or no donor and FLT3/ITD status.

OR indicates odds ratio; $\mathrm{Cl}$, confidence intervals; TRM, treatment-related mortality; RR, relapse risk; DFS, disease-free survival; OS, overall survival; - , not applicable. 


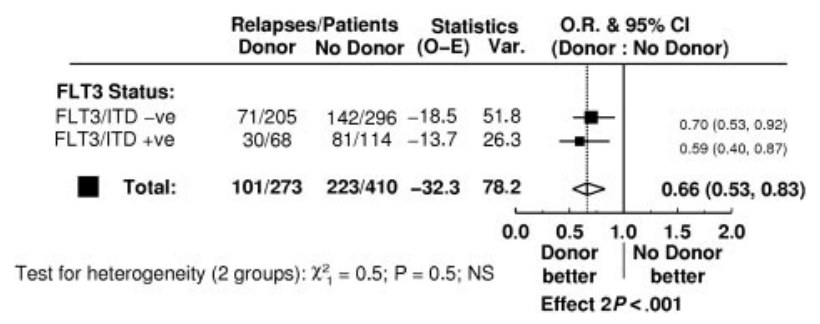

Figure 5. Relapse rate according to FLT3/ITD status in a donor-vs-no donor analysis of 683 patients.

of 750 or $1050 \mathrm{~Gy}$, or as 1440 cGy in 8 fractions, an undoubtedly myeloablative regimen.

As expected, the RR at 5 years in the total cohort of 141 autograft recipients was less than in those patients who did not receive consolidation with an autograft since, in order to have received a transplant, these patients had survived longer in first CR than some of the patients who did not receive a transplant who relapsed early. This is in accord with previously published results of valid randomized comparisons. ${ }^{17}$ However, a relatively increased risk of relapse associated with an FLT3/ITD remained in the patients who received an autograft, with the 5-year RR 56\% and $35 \%$, respectively, in patients with and without an FLT3/ITD. In addition, several studies have suggested that the prognosis is worse in those patients with a high relative level of mutant, irrespective of therapy received, ${ }^{21-23}$ and this situation also pertains following an autograft, with an actuarial RR of $75 \%$ in those patients who had at least 45\% mutant FLT3 at presentation (Figure 4B).

In this study, 186 patients were randomized to receive or not receive an autograft. Randomization to an autograft was associated with a similar reduction in the risk of relapse in both the FLT3/ITD $^{+}$and FLT3/ITD $^{-}$patients. Although there was unexpected evidence for possible heterogeneity in OS between transplantation and FLT3/ITD status, this may be a chance finding as there was no significant heterogeneity for relapse, and may be related to the very poor outcome seen in the small group $(n=26)$ of FLT3/ITD $^{+}$patients allocated to no autograft, with better outcome observed in other FLT3/ITD ${ }^{+}$patients who did not receive a transplant (for example, OS of $8 \%$ compared with $29 \%$ in the entire cohort that did not receive a transplant). Therefore, considering all the data on patients receiving cyclophosphamide and total body irradiation autografts, we conclude that this form of dose escalation does not overcome the resistance to chemoradiotherapy in the leukemic stem cells, and the FLT3 status cannot be used as a parameter on which to determine whether a patient should receive such an autograft.

The data on recipients of an allograft is rather less clear. In apparent contrast to patients receiving either no transplant or an autograft, in the allograft recipients there was an almost identical relapse rate in patients with and without an FLT3/ITD (Figure 4C), raising the possibility that a greater benefit had been seen in the FLT3/ITD $^{+}$patients. However, this analysis may be subject to both bias, due to selection of patients for SCT and samples available for analysis, and the play of chance since the numbers are very small and the $\mathrm{CI}$ is compatible with a similar OR for relapse as the patients who did not receive an allograft (Figure 3A). In the more statistically robust donor-versus-no donor analysis, the reduction in risk of relapse associated with having a donor, and thus the opportunity to have an allograft, was similar in both the FLT3/ ITD $^{+}$and FLT3/ITD ${ }^{-}$patients (Figure 5). This study therefore provides no good evidence that an allograft overcomes the chemoresistance/radioresistance inherent in FLT3/ITD ${ }^{+}$leukemic blasts. Consequently, outside of the context of a clinical trial, the presence of an FLT3/ITD should not be factored into the decisionmaking process as to whether any particular patient should receive an allograft.

These data are consistent with the impact of other prognostic factors such as the karyotype, which is influential whichever form of consolidation treatment is used. Results from the UK MRC AML10 and 12 trials have demonstrated that even though overall survival may not be improved, autologous or allogeneic transplantation reduced the relapse risk of every prognostic subgroup. ${ }^{2,15,17}$ The present study shows that this is also the case with respect to FLT3/ITD status. It confirms the poor prognosis of FLT3/ITD ${ }^{+}$ patients and does not substantiate the hypothesis that they might preferentially benefit from any form of transplantation consolidation. It must be acknowledged, however, that although this is the largest study evaluating FLT3/ITD status in patients who received a transplant, in order to confidently exclude heterogeneity in the response to different modalities of consolidation therapy, a much larger study incorporating in excess of 3000 non-APL AML patients would be required. The clinical management implications of answering this question are of such sufficient importance that a multigroup meta-analysis is now justified.

\section{Acknowledgment}

We are grateful to the clinical investigators who entered and managed patients in these 2 trials.

\section{References}

1. Hann IM, Stevens RF, Goldstone AH, et al. Randomized comparison of DAT versus ADE as induction chemotherapy in children and younger adults with acute myeloid leukemia: results of the Medical Research Council's 10th AML trial (MRC AML10). Adult and Childhood Leukaemia Working Parties of the Medical Research Council. Blood. 1997;89:2311-2318.

2. Burnett AK, Wheatley $K$, Goldstone $A H$, et al. MRC AML12: a comparison of ADE vs MAE and S-DAT vs H-DAT \pm retinoic acid for induction and four vs five total courses using chemotherapy or stem cell transplant in consolidation, in 3459 patients under 60 years with AML [abstract]. Blood. 2002; $100: 155 a$

3. Mayer RJ, Davis RB, Schiffer CA, et al. Intensive postremission chemotherapy in adults with acute myeloid leukemia: Cancer and Leukemia Group B. N Engl J Med. 1994;331:896-903.

4. Burnett AK. Treatment of acute myeloid leukaemia in younger patients. Best Pract Res Clin Haematol. 2001;14:95-118.

5. Gray R, Wheatley K. How to avoid bias when comparing bone marrow transplantation with chemotherapy. Bone Marrow Transplant. 1991; 7(Suppl 3):9-12.

6. Wheatley K. Current controversies: which patients with acute myeloid leukaemia should receive a bone marrow transplantation?-a statistician's view. Br J Haematol. 2002;118:351-356.

7. Harousseau JL, Cahn JY, Pignon B, et al. Comparison of autologous bone marrow transplantation and intensive chemotherapy as postremission therapy in adult acute myeloid leukemia: The
Groupe Ouest Est Leucemies Aigues Myeloblastiques (GOELAM). Blood. 1997;90:2978-2986.

8. Keating $S$, de Witte $T$, Suciu $S$, et al. The influence of HLA-matched sibling donor availability on treatment outcome for patients with AML: an analysis of the AML 8A study of the EORTC Leukaemia Cooperative Group and GIMEMA. European Organization for Research and Treatment of Cancer. Gruppo Italiano Malattie Ematologiche Maligne dell'Adulto. Br J Haematol. 1998;102: 1344-1353.

9. Cassileth PA, Harrington DP, Appelbaum FR, et al. Chemotherapy compared with autologous or allogeneic bone marrow transplantation in the management of acute myeloid leukemia in first remission. N Engl J Med. 1998;339:1649-1656.

10. Burnett AK, Wheatley K, Goldstone AH, et al. The value of allogeneic bone marrow transplant in 
From www.bloodjournal.org at UCL Library Services on August 20, 2008. For personal use only.

patients with acute myeloid leukaemia at differing risk of relapse: results of the UK MRC AML 10 trial. Br J Haematol. 2002;118:385-400.

11. Ferrant A, Labopin M, Frassoni F, et al. Karyotype in acute myeloblastic leukemia: prognostic significance for bone marrow transplantation in first remission: a European Group for Blood and Marrow Transplantation study. Acute Leukemia Working Party of the European Group for Blood and Marrow Transplantation (EBMT). Blood. 1997;90: 2931-2938.

12. Gale RP, Horowitz MM, Weiner RS, et al. Impact of cytogenetic abnormalities on outcome of bone marrow transplants in acute myelogenous leukemia in first remission. Bone Marrow Transplant. 1995; 16:203-208

13. Grimwade D, Walker H, Oliver F, et al. The importance of diagnostic cytogenetics on outcome in AML: analysis of 1,612 patients entered into the MRC AML 10 trial. The Medical Research Council Adult and Children's Leukaemia Working Parties. Blood. 1998;92:2322-2333.

14. Slovak ML, Kopecky KJ, Cassileth PA, et al. Karyotypic analysis predicts outcome of pre- remission and postremission therapy in adult acute myeloid leukemia: a Southwest Oncology Group/Eastern Cooperative Oncology Group Study. Blood. 2000;96:4075-4083.

15. Burnett AK, Wheatley K, Stevens R, et al. Further data to question the use of alloBMT in AML CR1 in addition to intensive chemotherapy: the MRC experience in 715 patients under 44 years with donors available [abstract]. Blood. 2002;100:74a

16. Suciu S, Mandelli F, de Witte T, et al. Allogeneic compared with autologous stem cell transplantation in the treatment of patients younger than 46 years with acute myeloid leukemia (AML) in first complete remission (CR1): an intention-to-treat analysis of the EORTC/GIMEMAAML-10 trial. Blood. 2003;102:1232-1240.

17. Burnett AK. Current controversies: which patients with acute myeloid leukaemia should receive a bone marrow transplantation?-an adult treater's view. Br J Haematol. 2002;118:357-364.

18. Kottaridis PD, Gale RE, Linch DC. Flt3 mutations and leukaemia. Br J Haematol. 2003;122:523538.

19. Stirewalt DL, Radich JP. The role of FLT3 in haematopoietic malignancies. Nat Rev Cancer. 2003;3:650-665.

20. Levis M, Small D. FLT3: ITDoes matter in leukemia. Leukemia. 2003;17:1738-1752.

21. Kottaridis PD, Gale RE, Frew ME, et al. The presence of a FLT3 internal tandem duplication in patients with acute myeloid leukemia (AML) adds important prognostic information to cytogenetic risk group and response to the first cycle of chemotherapy: analysis of 854 patients from the United Kingdom Medical Research Council AML 10 and 12 trials. Blood. 2001;98:1752-1759.

22. Whitman SP, Archer KJ, Feng L, et al. Absence of the wild-type allele predicts poor prognosis in adult de novo acute myeloid leukemia with normal cytogenetics and the internal tandem duplication of FLT3: a cancer and leukemia group B study. Cancer Res. 2001;61:7233-7239.

23. Thiede C, Steudel C, Mohr B, et al. Analysis of FLT3-activating mutations in 979 patients with acute myelogenous leukemia: association with FAB subtypes and identification of subgroups with poor prognosis. Blood. 2002;99:43254335. 